\title{
On the (Mis)Use of Wealth as a Proxy FOR Risk AVERSION
}

\author{
Marc F. Bellemare and Zachary S. Brown
}

\begin{abstract}
Tests of risk sharing in the contracting literature often rely on wealth as a proxy for risk aversion. The intuition behind these tests is that since contract choice is monotonic in the coefficients of risk aversion, which are themselves assumed monotonic in wealth, the effect of a change in wealth on contract choice is clearly identified. We show that tests of risk sharing relying on wealth as a proxy for risk aversion are identified only insofar as the econometrician is willing to assume that (a) the principal is risk neutral or her preferences exhibit constant absolute risk aversion (CARA); and (b) the agent is risk neutral.
\end{abstract}

Key words: contract theory, empirical tests, principal-agent models, risk aversion, risk sharing.

JEL codes: C12, D86, G32, Q19.

Empirical tests of risk sharing in the agricultural contracting literature often rely on wealth as a proxy for risk aversion. The intuition behind such tests is that since risk sharing (i.e., contract choice) is monotonic in the coefficients of absolute and relative risk aversion of the principal and the agent, and since these coefficients are themselves assumed to be monotonic in wealth, there is no harm in using the wealth of either party as a proxy for their risk preferences. ${ }^{1}$

Intuitively, and leaving aside for a moment the distinction between absolute and relative risk aversion, if one assumes that risk aversion is decreasing in wealth, both the principal and the agent should bear more risk as their wealth increases. If one assumes instead that risk aversion is constant in wealth, the amount of risk borne by both the principal and the agent should remain unchanged as their wealth

Marc F. Bellemare is an assistant professor at the Sanford Schoo of Public Policy, Duke University, Durham, NC; Zachary S. Brown is Ph.D. candidate at the Nicholas School of the Environment, Duke University, Durham, NC. We thank Jeff Dorfman and two anonymous referees for excellent comments and suggestions. We are also grateful to Chris Barrett, Catherine Guirkinger, Jaclyn Kropp, Gabriel Power, and the participants at the 2009 SCC-76 "Economics and Management of Risk in Agriculture and Natural Resources" conference for useful comments. Finally, we thank Rachel Kranton, Curtis Taylor, and Steve Wu for helpful conversations. All remaining errors are ours.

1 The terms "degree of risk sharing" and "contract choice" are used interchangeably throughout this article, given that in the literature on contract theory, the principal's choice of which contract to offer the agent is generally identical to determining how much risk the agent should bear. increases. And if one assumes that risk aversion is increasing in wealth, both the principal and the agent should bear less risk as their wealth increases. ${ }^{2}$ For example, in the literature on agrarian contracts, it is common to regress contract choice (i.e., sharecropping or fixed rent) on the wealth levels of the principal and the agent and to expect to find support in favor of the hypothesis that as principal (agent) wealth increases, a sharecropping (fixed rent) contract becomes more likely because the principal (agent) becomes more willing to take on production risk (Laffont and Matoussi 1995; Ackerberg and Botticini 2000, 2002; Dubois 2002; and Fukunaga and Huffman 2009). ${ }^{3}$

Given the frequent use of wealth as a proxy for risk aversion in testing for risk sharing in the agricultural contracting literature, this article characterizes how optimal risk sharing varies within a contract as the wealth levels of the principal and the agent vary. ${ }^{4}$ Consistent with the intuition underlying several tests of risk sharing, when the principal is risk neutral

\footnotetext{
2 In practice, applied contract theorists have often assumed that preferences exhibit decreasing absolute risk aversion, in accordance with Arrow's (1971) hypothesis about the shape of absolute risk aversion. Arrow (1971) had also hypothesized that relative risk aversion is increasing in wealth. Holt and Laury (2002) found experimental support for both hypotheses.

${ }^{3}$ The use of wealth as a proxy for risk aversion is not exclusive to agricultural economics. See Becker (2006) for an application to executive compensation.

${ }^{4}$ For risk sharing in other contexts (e.g., within a social network or a village), see Townsend (1994), Ligon (1998), Fafchamps and Lund (2003), Fafchamps and Gubert (2007), and Dubois, Jullien, and Magnac (2008), among others.
} 
or her preferences exhibit constant absolute risk aversion (CARA), a change in her wealth level has no effect on risk sharing. Likewise, when the agent is risk neutral, a change in his wealth has no effect on risk sharing. The contribution of this article is to illustrate how in other cases, the direction of the change in risk sharing is unidentified. ${ }^{5}$ While it is possible that the test of risk sharing is consistent with intuition in such cases, this cannot be shown without imposing further structure on the principal-agent problem.

In other words, except under very restrictive assumptions, the theory cannot generate hypotheses on whether the degree of risk sharing is affected positively or negatively by wealth changes, and a simple simulation-based example shows that risk sharing can change non-monotonically as wealth changes. So while several empirical studies find statistically significant linear effects of wealth on risk sharing, such results are likely the product of restrictions imposed by the econometrician (e.g., the linearity of risk sharing in wealth levels) rather than that of robust patterns of behavior. Consequently, any test of contractual risk sharing that relies on wealth as a proxy for risk aversion will be useful only insofar as the econometrician is willing to either: (a) assume that the principal is risk neutral or that her preferences exhibit CARA and that the agent is risk neutral; or (b) impose a great deal of structure on the principal-agent problem.

The intuition behind our main result is as follows. Whereas changing wealth is usually thought to affect only risk aversion, incorporating ex ante wealth levels as arguments in the respective utility functions of the principal and the agent implies that wealth changes affect not only risk aversion, but also the marginal utilities that determine the optimal contract. ${ }^{6}$ The trade-off between the degree of risk aversion and the marginal utility of either party is thus what leads to an ambiguous effect on risk sharing.

The rest of this article is organized as follows. First, a general principal-agent model is set up incorporating the ex ante wealth levels of the principal and the agent in their respective utility functions. Next, the effects of exogenous

\footnotetext{
${ }^{5}$ In this article, an empirical test is said to be identified if and only if the theory yields a clear testable implication.

6 Incorporating wealth as an argument in the utility function is necessary in this case, both because the study of wealth effects requires it, and because expected utility is defined over final wealth, i.e., not just over the income derived from the contract or gamble under study.
}

changes in wealth on risk sharing are presented and discussed for both absolute and relative risk aversion. A simulation-based example follows, in which a test of risk sharing relying on wealth as a proxy for risk aversion would be unidentified in the simple case where the principal is risk neutral, the agent is risk averse, and preferences exhibit CARA. We conclude by briefly discussing potential solutions.

\section{A Principal-Agent Model with Exogenous Wealth}

Consider the following hidden action (i.e., ex ante moral hazard) principal-agent model (Bolton and Dewatripont 2005). A principal whose utility function is $V(\cdot)$, with $V^{\prime}>0$ and $V^{\prime \prime} \leq 0$, contracts with an agent whose utility function is $U(\cdot)$, with $U^{\prime}>0$ and $U^{\prime \prime} \leq 0 .{ }^{7}$ The principal hires the agent to produce output $q \in$ $[q, \bar{q}]$. Output is stochastic, and its realization depends on the effort of the agent, $e \in E$, which is unobserved by the principal. Output and effort are linked through the conditional probability density function $f(q \mid e)$, which describes the likelihood of observing output level $q$ given effort level $e$. In what follows, the price $p$ of output $q$ is normalized and set equal to one so as to alleviate notation.

The agent's payoff from accepting the contract offered by the principal is assumed additively separable in the utility derived from the contract and in the cost of effort, which is represented by the twice continuously differentiable function $\psi(e)$, with $\psi^{\prime}>0$ and $\psi^{\prime \prime}>0$. The principal solves her maximization problem by offering a contract $w(q)$ to the agent.

The ex ante wealth levels of the principal and the agent are respectively $z_{p}$ and $z_{a}$, so that the utility of the principal is defined over $\tilde{z}_{p}=z_{p}+q-w(q)$, i.e., her ex ante wealth level plus her payoff from the contract, and the utility of the agent is defined over $\tilde{z}_{a}=z_{a}+w(q)$, i.e., his ex ante wealth level plus his payoff from the contract. The ex post levels of utility of the principal and the agent are thus $V\left(\tilde{z}_{p}\right)=V\left[z_{p}+q-w(q)\right]$ and $U\left(\tilde{z}_{a}\right)=U\left[z_{a}+\right.$ $w(q)]$, respectively. In most of the literature, the ex ante wealth levels are implicitly set equal to zero to focus on the contract itself,

\footnotetext{
${ }^{7}$ We allow for the possibility that both the principal and the agent are risk averse based on Holt and Laury's (2002) conclusion that "clear evidence for risk aversion, even with low stakes, suggests the potential danger of analyzing behavior under the simplifying assumption of risk neutrality."
} 
but our focus on wealth effects requires that they be incorporated in the utility function, as in Menezes and Hanson (1970) and Zeckhauser and Keeler (1970), and more recently in Meyer and Meyer (2005) and Guo and Ou-Yang (2006). ${ }^{8}$ Finally, the agent's reservation utility is $\bar{U}=U\left(z_{a}\right)$, which accounts for the fact that the agent receives his ex ante wealth regardless of whether he participates in the contract or not.

The principal's problem is thus to

$$
\begin{aligned}
& \max _{\{w(q), e\}} \int_{\underline{q}}^{\bar{q}} V\left(\tilde{z}_{p}\right) f(q \mid e) d q \text { subject to } \\
& \int_{\underline{q}}^{\bar{q}} U\left(\tilde{z}_{a}\right) f(q \mid e) d q-\psi(e) \\
& \quad \geq U\left(z_{a}\right), \text { and }(\mathrm{IR}) \\
& e \in \underset{\hat{e} \in E}{\arg \max }\left[\int_{\underline{q}}^{\bar{q}} U\left(\tilde{z}_{a}\right) f(q \mid \hat{e}) d q-\psi(\hat{e})\right]
\end{aligned}
$$

where IR denotes the agent's individual rationality constraint and IC denotes his incentive compatibility constraint. Assume that the agent's maximization problem has a unique solution and that both the monotone likelihood ratio property (MLRP) and the convexity of the distribution function condition (CDFC) hold. One can then apply the firstorder approach (Rogerson 1985) and replace the incentive compatibility constraint with its first-order condition (FOC), so that equation (3) becomes

$$
\int_{\underline{q}}^{\bar{q}} U\left(\tilde{z}_{a}\right) f_{e}(q \mid e) d q-\psi^{\prime}(e)=0 .
$$

The standard way of solving such problems is by backward induction: assuming the principal already knows which effort level she wants to implement, she will then design a contract which most efficiently implements this effort level, subject to the agent's IR and IC' constraints. This yields a function for the efficient contract defined over the space of implementable effort levels; the induction

\footnotetext{
${ }^{8}$ Excluding wealth from the utility functions of the principal and the agent would essentially be equivalent to assuming a reference point-based framework similar to prospect theory (Kahneman and Tversky 1979), but without loss aversion.
}

step derives the optimal $\{w(q), e\}$ pair within this space.

Setting up the Lagrangian and solving by differentiating inside the integral sign with respect to $w(q)$ yields

$$
\frac{V^{\prime}\left(\tilde{z}_{p}\right)}{U^{\prime}\left(\tilde{z}_{a}\right)}=\lambda\left(z_{a}, z_{p}\right)+\mu\left(z_{a}, z_{p}\right) \frac{f_{e}(q \mid e)}{f(q \mid e)}
$$

where $f_{e}(q \mid e)=\frac{\partial f(q \mid e)}{\partial e}$ and $\lambda\left(z_{a}, z_{p}\right)$ and $\mu\left(z_{a}, z_{p}\right)$ are the multipliers on the agent's IR and IC' constraints, respectively. A standard result (see online appendix) is that these multipliers are independent of $q$. In general, however, they will depend on ex ante wealth levels.

Differentiating equation (5) with respect to $q$ yields

$$
\begin{aligned}
& \frac{d w\left(q, z_{a}, z_{p}\right)}{d q} \\
& \left\{\mu\left(z_{a}, z_{p}\right) U^{\prime 2}\left(\tilde{z}_{a}\right) \frac{d}{d q}\left[\frac{f_{e}(q \mid e)}{f(q \mid e)}\right]\right. \\
& =\frac{\left.-V^{\prime \prime}\left(\tilde{z}_{p}\right) U^{\prime}\left(\tilde{z}_{a}\right)\right\}}{\left[-V^{\prime \prime}\left(\tilde{z}_{p}\right) U^{\prime}\left(\tilde{z}_{a}\right)-U^{\prime \prime}\left(\tilde{z}_{a}\right) V^{\prime}\left(\tilde{z}_{p}\right)\right]}
\end{aligned}
$$

which is the slope of the contract with respect to output, and which characterizes optimal risk sharing. Let $w_{q}\left(q, z_{a} z_{p}\right)=\frac{d w\left(q, z_{a}, z_{p}\right)}{d q}$ and $\theta=$ $\frac{d}{d q}\left[\frac{f_{e}(q \mid e)}{f(q \mid e)}\right]$. Then, given that the MLRP holds, $\theta>0$, and so $w_{q}\left(q, z_{a} z_{p}\right)>0$. That is, the agent's compensation increases as output $q$ increases. But since $w_{q}\left(q, z_{a}, z_{p}\right)$ measures the sensitivity of the agent's compensation $w\left(q, z_{a}, z_{p}\right)$ to changes in output $q, w_{q}\left(q, z_{a}, z_{p}\right)$ also measures the degree of risk sharing. This is most easily understood within a linear contract, i.e., a contract of the form $w(q)=a q+b$, where $w_{q}=a$ is the share of the risk that accrues to the agent, and $(1-a)$ is the share of the risk that accrues to the principal. For example, in land tenancy contracts, if $a=0$, the landlord pays the tenant a fixed wage; if $a \in(0,1)$, the landlord and the tenant sign a sharecropping contract; and if $a=1$, the tenant pays the landlord a fixed rent.

Omitting arguments for clarity and dividing each term of the numerator and the denominator in equation (6) by $U^{\prime}\left(\tilde{z}_{a}\right) V^{\prime}\left(\tilde{z}_{p}\right)$ 
yields

$$
w_{q}=\frac{\frac{\mu U^{\prime}\left(\tilde{z}_{a}\right) \theta}{V^{\prime}\left(\tilde{z}_{p}\right)}+A_{p}}{A_{p}+A_{a}}
$$

where $A_{p}=-\frac{V^{\prime \prime}\left(\tilde{z}_{p}\right)}{V^{\prime}\left(\tilde{z}_{p}\right)}$ and $A_{a}=-\frac{U^{\prime \prime}\left(\tilde{z}_{a}\right)}{U^{\prime}\left(\tilde{z}_{a}\right)}$ are the Arrow-Pratt coefficients of absolute risk aversion of the principal and the agent, respectively. Likewise, multiplying each term of the numerator and the denominator in equation (7) by $\tilde{z}_{a} \tilde{z}_{p}$ yields

$$
w_{q}=\frac{\frac{\mu U^{\prime}\left(\tilde{z}_{a}\right) \theta \tilde{z}_{a} \tilde{z}_{p}}{V^{\prime}\left(\tilde{z}_{p}\right)}+R_{p} \tilde{z}_{a}}{R_{p} \tilde{z}_{a}+R_{a} \tilde{z}_{p}}
$$

where $R_{p}=-\frac{V^{\prime \prime}\left(\tilde{z}_{p}\right)}{V^{\prime}\left(\tilde{z}_{p}\right)} \tilde{z}_{p}$ and $R_{a}=-\frac{U^{\prime \prime}\left(\tilde{z}_{a}\right)}{U^{\prime}\left(\tilde{z}_{)}\right)} \tilde{z}_{a}$ are the Arrow-Pratt coefficients of relative risk aversion of the principal and the agent, respectively.

What has so far led applied contract theorists to use wealth as a proxy for risk aversion is (a) the omission of the ex ante wealth levels from the utility functions; in conjunction with (b) the fact that $\frac{\partial w_{q}}{\partial A_{p}} \geq 0$ and $\frac{\partial w_{q}}{\partial A_{q}}<0$ if one considers absolute risk aversion or $\frac{\partial w_{q}}{\partial R_{P}} \geq 0$ and $\frac{\partial w_{q}}{\partial R_{a}}<0$ if one considers relative risk aversion. ${ }^{9}$

In that case, it is only natural to assume that using wealth as a proxy for either absolute or relative risk aversion will lead to testing the null hypothesis of no risk sharing against the right alternative hypothesis, since coefficients of absolute (relative) risk aversion are monotonic in wealth under decreasing absolute or relative risk aversion (DARA or DRRA), constant absolute or relative risk aversion (CARA or CRRA), and increasing absolute or relative risk aversion (IARA or IRRA). The next section explores the effect of changing the wealth levels of the principal and the agent on risk sharing.

\section{The Effects of Changes in Wealth on Risk Sharing}

In a slight abuse of notation, let $\mu_{a}=\frac{\partial \mu}{\partial z_{a}}$, $\mu_{p}=\frac{\partial \mu}{\partial z_{p}}, w_{a}=\frac{\partial w}{\partial z_{a}}$, and $w_{p}=\frac{\partial w}{\partial z_{p}}$. Moreover,

\footnotetext{
${ }^{9}$ On the principal's side, the results for both absolute and relative risk aversion follow from the fact that $w_{q} \leq 1$,i.e., the degree of risk sharing cannot exceed one, since the agent cannot bear more risk than there is within the contract. See Bellemare (2008) for a formal proof.
}

let $P_{p}=-\frac{V^{\prime \prime \prime}\left(\tilde{z}_{p}\right)}{V^{\prime \prime}\left(\tilde{z}_{p}\right)}$ and $P_{a}=-\frac{U^{\prime \prime \prime}\left(\tilde{z}_{a}\right)}{U^{\prime \prime}\left(\tilde{z}_{a}\right)}$ respectively denote the coefficients of absolute prudence of the principal and the agent (Kimball 1990), i.e., their respective attitudes with respect to precautionary savings. Given equations (7) and (8), tables 1 and 2 show the effect of changing the wealth levels of the principal and the agent on risk sharing when considering absolute and relative risk aversion, respectively. ${ }^{10}$ Based on the results in tables 1 and 2, the following results can now be stated.

Proposition 1. If the principal is risk neutral or if her preferences exhibit CARA, $\partial w_{q} /$ $\partial z_{p}=0$.

Proof. If the principal is risk neutral, her utility function $V\left(\tilde{z}_{p}\right)$ can without loss of generality be written as

$$
V\left(\tilde{z}_{p}\right)=z_{p}+q-w(q)
$$

in which case her maximization problem becomes

$$
\max _{\{w(q), e\}} z_{p}+\int_{\underline{q}}^{\bar{q}}[q-w(q)] f(q \mid e) d q
$$

subject to the IR and IC' constraints defined by equations (2) and (4). In this case, any change in $z_{p}$ implies an affine transformation of $V(\cdot)$, leading to a strictly monotonic transformation of the principal's objective function and leaving the optimal contract unchanged (see proposition 6.B.2. in Mas-Colell, Whinston, and Green 1995, p. 173). That is, for all $z_{p} \in R$, we have the equivalence:

$$
\begin{gathered}
\max _{\{w(q), e\}} z_{p}+\int_{\underline{q}}^{\bar{q}}[q-w(q)] f(q \mid e) d q \\
\Leftrightarrow \max _{\{w(q), e\}} \int_{\underline{q}}^{\bar{q}}[q-w(q)] f(q \mid e) d q .
\end{gathered}
$$

Likewise, if the principal's preferences exhibit CARA, her utility function $V\left(\tilde{z}_{p}\right)$ can without

\footnotetext{
${ }^{10}$ The results of the empirical test are the same whether one considers absolute or relative risk aversion, since equation (8) is obtained by multiplying equation (7) by one. Table 2 shows the results for relative risk aversion only for completeness.
} 
Table 1. Change in Risk Sharing as Wealth Levels Change: Absolute Risk Aversion

$$
\frac{\partial w_{q}}{\partial z_{a}}=\frac{\left\{\frac{\mu_{a} U^{\prime} \theta}{V^{\prime}}-\frac{\mu U^{\prime} \theta}{V^{\prime}}\left[A_{a}+w_{a}\left(A_{p}+A_{a}\right)\right]-A_{p}\left(A_{p}-P_{P}\right) w_{a}\right\}}{\left(A_{p}+A_{a}\right)}-\frac{\left\{A_{a}\left(A_{a}-P_{a}\right)\left(1+w_{a}\right)-A_{p}\left(A_{p}-P_{p}\right) w_{a}\right\}\left(\frac{\mu U^{\prime} \theta}{V^{\prime}}+A_{p}\right)}{\left(A_{p}+A_{a}\right)^{2}}
$$

$$
\frac{\partial w_{q}}{\partial z_{p}}=\frac{\left\{\frac{\mu_{p} U^{\prime} \theta}{V^{\prime}}-\frac{\mu U^{\prime} \theta}{V^{\prime}}\left[A_{p}+w_{p}\left(A_{a}-A_{p}\right)\right]+A_{p}\left(A_{p}-P_{P}\right)\left(1-w_{p}\right)\right\}}{\left(A_{p}+A_{a}\right)}-\frac{\left\{A_{p}\left(A_{p}-P_{p}\right)\left(1-w_{p}\right)+A_{a}\left(A_{a}-P_{a}\right) w_{p}\right\}\left(\frac{\mu U^{\prime} \theta}{V^{\prime}}+A_{p}\right)}{\left(A_{p}+A_{a}\right)^{2}}
$$

\section{Table 2. Change in Risk Sharing as Wealth Levels Change: Relative Risk Aversion}

$$
\begin{aligned}
& \frac{\partial w_{q}}{\partial z_{a}}=\frac{\left\{\frac{\mu_{a} U^{\prime} \theta \tilde{z}_{a} \tilde{z}_{p}}{V^{\prime}}-\mu \theta \tilde{z}_{a} \tilde{z}_{p}\left[A_{a}+w_{a}\left(A_{p}+A_{a}\right)\right]+\frac{\mu U^{\prime}\left(1+w_{a}\right) \theta \tilde{z}_{p}}{V^{\prime}}-\frac{\mu U^{\prime} \theta \tilde{z}_{a} w_{a}}{V^{\prime}}-A_{p}\left(A_{p}-P_{P}\right) w_{a} \tilde{z}_{a} \tilde{z}_{p}+-A_{p} w_{a} \tilde{z}_{a}+A_{p} \tilde{z}_{p}\left(1+w_{a}\right)\right\}}{\left(R_{a} \tilde{z}_{a} \tilde{z}_{p}\right)} \\
& -\frac{\left\{-A_{p}\left(A_{p}-P_{P}\right) w_{a} \tilde{z}_{a} \tilde{z}_{p}+-A_{p} w_{a} \tilde{z}_{a}+A_{p} \tilde{z}_{p}\left(1+w_{a}\right)+A_{a}\left(A_{a}-P_{a}\right)\left(1+w_{a}\right) \tilde{z}_{a} \tilde{z}_{p}+A_{a}\left(1+w_{a}\right) \tilde{z}_{p}+A_{a} \tilde{z}_{a} w_{a}\right\}\left(\frac{\mu U^{\prime} \theta \tilde{z}_{a} \tilde{z}_{p}}{V^{\prime}}+R_{p} \tilde{z}_{a}\right)}{\left(R_{p} \tilde{z}_{a}+R_{a} \tilde{z}_{p}\right)^{2}} \\
& \frac{\partial w_{q}}{\partial z_{p}}=\frac{\left\{\frac{\mu_{p} U^{\prime} \theta \tilde{z}_{a} \tilde{z}_{p}}{V^{\prime}}-\mu \theta \tilde{z}_{a} \tilde{z}_{p}\left[A_{p}+w_{p}\left(A_{a}-A_{p}\right)\right]+\frac{\mu U^{\prime} \theta w_{p} \tilde{z}_{p}}{V^{\prime}}+\frac{\mu U^{\prime} \theta \tilde{z}_{a}\left(1+w_{p}\right)}{V^{\prime}}+A_{p}\left(A_{p}-P_{P}\right)\left(1-w_{p}\right) \tilde{z}_{p} \tilde{z}_{a}+A_{p}\left(1-w_{p}\right) \tilde{z}_{a}+A_{p} \tilde{z}_{p} w_{p}\right\}}{\left(R_{p} \tilde{z}_{a}+R_{a} \tilde{z}_{p}\right)} \\
& \left\{A_{p}\left(A_{p}-P_{P}\right)\left(1-w_{p}\right) \tilde{z}_{p} \tilde{z}_{a}+A_{p}\left(1-w_{p}\right) \tilde{z}_{a}+A_{p} \tilde{z}_{p} w_{p}+A_{a}\left(A_{a}-P_{a}\right) w_{p} \tilde{z}_{a} \tilde{z}_{p}+A_{a} w_{p} \tilde{z}_{p}+A_{a} \tilde{z}_{a}\left(1-w_{p}\right)\right\}\left(\frac{\mu U^{\prime} \theta \tilde{z}_{a} \tilde{z}_{p}}{V^{\prime}}+R_{p} \tilde{z}_{a}\right) \\
& -\frac{\left(R_{p} \tilde{z}_{a}+R_{a} \tilde{z}_{p}\right)^{2}}{V^{2}}
\end{aligned}
$$


loss of generality be written as

$$
V\left(\tilde{z}_{p}\right)=-\exp \left\{-A_{p}\left[z_{p}+q-w(q)\right]\right\} .
$$

In this case, note that $A_{p}$ does not depend on $z_{p}$ because of the CARA assumption. The principal's objective function becomes

$$
\begin{aligned}
& \max _{\{w(q), e\}} \int_{\underline{q}}^{\bar{q}} \exp \left\{-A_{p} z_{p}\right\} \\
& \quad \times \exp \left\{-A_{p}[q-w(q)]\right\} f(q \mid e) d q
\end{aligned}
$$

subject to the IR and IC' constraints defined by equations (2) and (4). But this problem can be rewritten as

$$
\begin{aligned}
& \max _{\{w(q), e\}} \exp \left\{-A_{p} z_{p}\right\} \\
& \quad \times \int_{\underline{q}}^{\bar{q}} \exp \left\{-A_{p}[q-w(q)]\right\} f(q \mid e) d q
\end{aligned}
$$

subject again to the IR and IC' constraints defined by equations (2) and (4). In this case, a marginal change in $z_{p}$ also implies an affine transformation of $V(\cdot)$, allowing us to invoke the same result used above. That is, for all $z_{p} \in R$, we have established the equivalence

$$
\begin{aligned}
& \max _{\{w(q), e\}} \exp \left\{-A_{p} z_{p}\right\} \\
& \quad \times \int_{\underline{q}}^{\bar{q}} \exp \left\{-A_{p}[q-w(q)]\right\} f(q \mid e) d q \\
& \Leftrightarrow \max _{\{w(q), e\}} \int_{\underline{q}}^{\bar{q}} \exp \left\{-A_{p}[q-w(q)]\right\} \\
& \quad \times f(q \mid e) d q .
\end{aligned}
$$

The online appendix provides a less elegant albeit more intuitive explanation of this result.

The results in the proposition are important insofar as they identify how certain utilitytheoretic assumptions can imply restrictions on the contract as it depends on principal wealth. The econometric test following from this theory is powerful in the sense that we can jointly reject the hypotheses of a risk neutral or a CARA principal by establishing that the wealth of the principal has direct explanatory power in the contract. The proposition, however, provides no help in identifying what an alternative preference structure for the principal might be. A similar examination of agent wealth effects yields the following.

Proposition 2. If the agent is risk neutral, $\partial w_{q} / \partial z_{a}=0$.

Proof. In addition to the fact that $\int_{q}^{\bar{q}} f(q \mid e) d q=1$, the proof rests on the fact that $\int_{q}^{\overline{\bar{q}}} f_{e}(q \mid e) d q=0$. If the agent is risk neutral, his utility function $U\left(\tilde{z}_{a}\right)$ is such that

$$
U\left(\tilde{z}_{a}\right)=z_{a}+w(q)
$$

We want to show that if the IR and IC' constraints bind for some $z_{a}$, then they are satisfied for any $z_{a}$. Thus, suppose that the constraints are satisfied for $z_{a}=0$, in which case we have

$$
\int_{\underline{q}}^{\bar{q}} w(q) f(q \mid e) d q-\psi(e)-U(0)=0
$$

and

$$
\int_{\underline{q}}^{\bar{q}} w(q) f_{e}(q \mid e) d q-\psi^{\prime}(e)=0 .
$$

Then, for $z_{a} \in R$, these constraints can be rewritten as

$$
\begin{aligned}
& z_{a} \int_{\underline{q}}^{\bar{q}} f(q \mid e) d q \\
& \quad+\int_{\underline{q}}^{\bar{q}} w(q) f(q \mid e) d q-\psi(e)=z_{a}
\end{aligned}
$$

and

$$
\begin{aligned}
& \text { (20) } \quad z_{a} \int_{\underline{q}}^{\bar{q}} f_{e}(q \mid e) d q \\
& \quad+\int_{\underline{q}}^{\bar{q}} w(q) f_{e}(q \mid e) d q-\psi^{\prime}(e)=0
\end{aligned}
$$

But then, since $\int_{\underline{q}}^{\bar{q}} f(q \mid e) d q=1$, the IR constraint becomes

(21) $\int_{\underline{q}}^{\bar{q}} w(q) f(q \mid e) d q-\psi(e)=0$ 
and since $\int_{\underline{q}}^{\bar{q}} f_{e}(q \mid e) d q=0$, the IC' constraint becomes

$$
\int_{\underline{q}}^{\bar{q}} w(q) f_{e}(q \mid e) d q-\psi^{\prime}(e)=0
$$

In which case agent wealth $z_{a}$ drops out of the problem, and so $\frac{\partial w_{q}}{\partial z_{a}}=0$.

The proofs of propositions 1 and 2 make it clear that these results hold whether or not we allow effort to readjust to its second-best optimum following a wealth change.

Beyond the highly restrictive situations circumscribed by propositions 1 and 2, it is difficult to draw any conclusions regarding whether wealth changes induce a positive or negative response in optimal risk sharing. A formal comparative statics exercise examining the relationship between $w_{q}$ the wealth levels of the principal and the agent (see online appendix) does not yield any empirically testable monotonicity result in the general case. Indeed, as we show in the next section, there are situations in which the contract is non-monotonic in wealth levels.

To summarize the empirical content of this section, we turn to the regression of interest in tests of risk sharing and discuss how our results relate to it. In general, empirical work on contracts is often aimed at studying the determinants of contract choice by estimating the equation

$$
y=\alpha+\beta_{p} z_{p}+\beta_{a} z_{a}+\gamma x+\varepsilon
$$

where $y \equiv w_{q}$, which captures contract choice or the degree of risk sharing, is the dependent variable; $x$ is a vector of relevant covariates; and $\beta_{p}$ and $\beta_{a}$ are the coefficients typically required to test the null hypothesis of no risk sharing, i.e., $H_{0}: \beta_{p}=0$ and $\beta_{a}=0$. This specific functional form, in which the degree of risk sharing is linearly projected on the wealth levels of the principal and the agent, arises from the belief that risk sharing is monotonic in the wealth levels of the principal and the agent, which the derivations above have shown to be generally misguided. Proposition 1 identifies two distinct types of preferences for the principal which lead to $\beta_{p}=0$, and proposition 2 identifies one formulation of agent preferences implying that $\beta_{a}=0$. Consequently, if one rejects $H_{0}$, one rejects the hypothesis that (a) the principal is risk neutral; (b) the principal's preferences exhibit CARA; and (c) the agent is risk neutral.

The following section provides a preliminary investigation of the general case illustrating the fundamental conclusion that, at the very least, higher-order terms in wealth should be included in regressions of the contract type on wealth measures.

\section{A Simulation-Based Example}

Propositions 1 and 2 circumscribe where the relationship (or lack thereof) between the degree of risk sharing and wealth levels is identified. Perhaps more interestingly, another highly restrictive case-that of an agent whose preferences exhibit CARA-does not allow identification of the relationship between the degree of risk sharing and agent wealth. This is because of the nature of the canonical principal-agent model and, in particular, the way the agent's cost of effort is formulated. This section, which presents results from a simple simulation of the model with an agent whose preferences exhibit CARA, reveals a clear non-monotonic relationship between the degree of risk sharing and agent wealth.

Consider a situation in which output is measured in the same units as wealth and in which there are only two possible levels of output, i.e., $q \in\{q, \bar{q}\}$. The probability of a high output is increasing and concave in agent effort $e$ and is denoted by $P(e)$, so that the probability of a low output is equal to $1-P(e)$. Both utility function as well as the functions characterizing the cost to the agent of implementing effort $e$ and his reservation utility are defined as in the second section. Solving for the constrained optimal output-contingent contract levels $\bar{w}$ and $\underline{w}$ yields

$$
\begin{aligned}
& \left(\underline{w}^{*}, \bar{w}^{*}\right) \\
& =\arg \max V\left[z_{p}+\bar{q}-\bar{w}\right] P[e(\underline{w}, \bar{w})] \\
& \quad+V\left[z_{p}+\underline{q}-\underline{w}\right]\{1-P[e(\underline{w}, \bar{w})]\}
\end{aligned}
$$

subject to the agent's IR and IC constraints, defined in this case as

$$
\begin{aligned}
& U\left(z_{a}+\bar{w}\right) P[e(\underline{w}, \bar{w})] \\
& \quad+U\left(z_{a}+\underline{w}\right)\{1-P[e(\underline{w}, \bar{w})\} \\
& \quad-\psi[e(\underline{w}, \bar{w})] \geq U\left(z_{a}\right)
\end{aligned}
$$


and

$$
\begin{aligned}
e(\underline{w}, \bar{w}) \in & \arg \max U\left(z_{a}+\bar{w}\right) P(e) \\
& +U\left(z_{a}+\underline{w}\right)[1-P(e)] \\
& -\psi(e) .
\end{aligned}
$$

Given equations (12) to (14), let $\frac{\Delta w}{\Delta q}=\frac{\bar{w}-\underline{w}}{\bar{q}-\underline{q}}$ (i.e., the discrete-outcome equivalent of $w_{q}$ above) measure risk sharing, or the principal's choice of contract. We now turn to characterizing risk sharing for the case where the utility functions of the principal and the agent exhibit CARA.

Table 3 characterizes the specific parameterization we adopt. To determine how changing the wealth of the agent should affect risk sharing, we numerically solve for $e(\underline{w}, \bar{w})$ given a contract $(\underline{w}, \bar{w})$ in the IC constraint and then substitute the optimal effort into the IR constraint and the principal's objective function. In other words, we solve by backward induction for the usual sub-game perfect Nash equilibrium in the principal-agent model. The order of the induction steps, however, is reversed from the usual procedure in which the optimal contract is solved given an effort level before solving for the optimal effort level. This approach effectively substitutes effort out of the problem using the IC constraint.

Figure 1 plots the IR constraint in the $(\underline{w}, \bar{w})$ space along with the iso-utility curves of the principal and the resulting tangency points corresponding to the optimal outcome in three different cases: (a) the baseline case in which $z_{a}=1.5$, depicted in the southwestern tangency point; (b) the case in which agent wealth is increased by $50 \%$, depicted in the northeastern tangency point; and (c) the suboptimal case in which the IR locus alone responds to the increase in agent wealth, depicted in the northwestern tangency point. Because the principal's utility is decreasing in the agent's

\section{Table 3. Simulation Parameterization}

$$
\begin{aligned}
& V\left(z_{p}\right)=-\exp \left\{-A_{p} z_{p}\right\} \\
& U\left(z_{a}\right)=-\exp \left\{-A_{a} z_{a}\right\} \\
& A_{p}=1 \\
& A_{a}=1 \\
& q=0 \\
& \overline{\bar{q}}=1 \\
& P(e)=1-\exp (-1.5 e) \\
& \psi(e)=0.005 e^{2} \\
& e \in[0, \infty)
\end{aligned}
$$

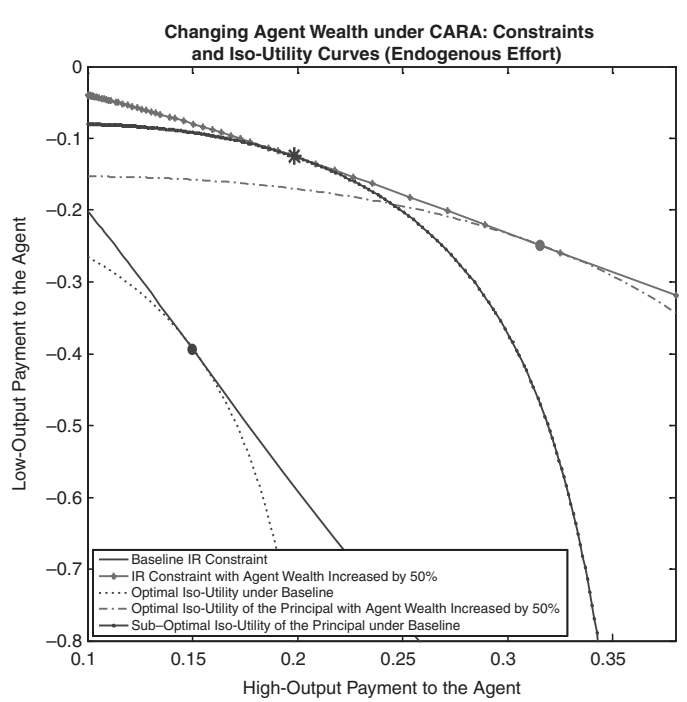

Figure 1. Individual rationality constraint and iso-utility curves of the principal under low and high outputs

payoff, points to the southwest of the contract space are preferred by the principal.

Figure 1 indicates that an increase in agent wealth has two distinct effects. The first effect, which we call the rationality effect, shifts the (binding) IR locus. Intuitively, this occurs because as the agent gets wealthier, the amount of baseline compensation he requires in order to accept the contract increases because his preferences are increasing in wealth and income, and it reflects both the direct impact of an increase in agent wealth as well as the indirect impact of a change in optimal effort. The second effect, which we call the incentive effect, is a shift in the iso-utility curves of the principal resulting from a change in expectations regarding output, which is itself the result of a change in the optimal effort of the agent. Intuitively, this occurs because as the agent gets wealthier, the substitution patterns between the increase in utility derived from wealth and income and the decrease in disutility derived from effort (alternatively, the increase in utility derived from leisure) change to reflect this. In general, a wealthier agent faces a stronger disincentive to exert effort in exchange for income. As a result, the net effect of an increase in agent wealth is to increase both state-contingent payments. Risk sharing, however, is determined by how increases in these payments compare with one another.

Indeed, separating out these effects leads to an interesting finding. Intuitively, one would expect a change in the agent's wealth to have 
no impact on the amount of risk borne by the agent if his preferences exhibit CARA. But as shown in figure 2, a $50 \%$ increase in agent wealth yields a $4 \%$ increase in risk sharing, from 0.543 to 0.563 . That is, the high-output payment increases more than the low-output payment. The figure also illustrates the risksharing impacts of the rationality effect in isolation. As discussed above, the rationality effect implies that an increase in agent wealth leads to the principal paying the agent a higher compensation to attain a higher level of reservation utility. In the absence of an incentive effect (i.e., a simultaneous shift in the principal's isoutility curves), however, the low-output payment increases more than the high-output payment, yielding a decrease in risk sharing to 0.323. In isolating this effect, it is as if the principal is responding to the agent's requirement to meet a higher reservation utility, but not recognizing how the agent's decreased effort leads to changes in her own expected utility. As we would expect, when the incentive effect is also accounted for, the principal finds it in her best interest to offer a more incentivized contract than would be the case accounting for the rationality effect alone.

But what we ultimately care about is the net effect of wealth changes on risk sharing, and the U-shaped relationship between the amount of risk borne by the agent and his wealth in figure 2 shows why relying on wealth as a proxy

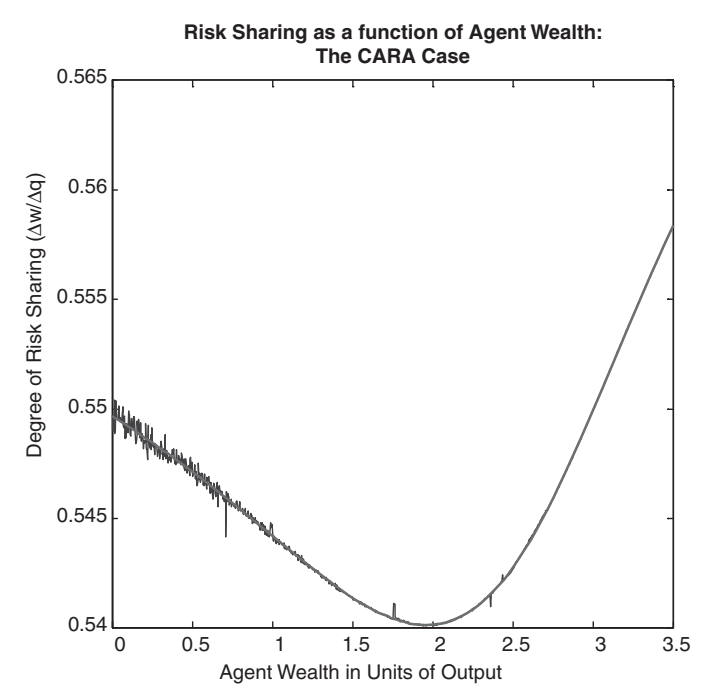

Note:The jagged thin vertical lines on the left-hand side of the figure are due to machine error in the numerical optimization routine used to solve the principal-agent problem described in the text. The thicker line smoothes out this machine error.

Figure 2. Risk sharing as a function of agent wealth under constant absolute risk aversion for risk aversion is generally not valid when studying the determinants of contract choice.

\section{Conclusion}

Given the frequent reliance on wealth as a proxy for risk aversion in the empirical contracting literature, this article has characterized how optimal risk sharing changes as the wealth levels of the principal and the agent vary under both absolute and relative risk aversion. Contrary to the intuition underlying several tests of risk sharing in the applied contracting literature, the direction of the change in optimal risk sharing as the wealth of either party changes is rarely identified.

More specifically, we have shown that the usual test can be used to reject (a) the risk neutrality of the principal; (b) that the principal's preferences exhibit CARA; and (c) the risk neutrality of the agent. In the simple case where the agent's preferences exhibit CARA, both our analytical derivations and our simulation-based example show that the effect of a change in wealth on risk sharing is non-monotonic and cannot be identified with a simple linear regression.

In order to properly conduct tests of risk sharing within a contract, then, two options are available to applied microeconomists. First, one can rely directly on coefficients of absolute risk aversion, although Lybbert and Just (2007) show that using estimated rather than experimentally derived coefficients of risk aversion can introduce a significant amount of spurious correlation between wealth and risk aversion. Second, one can choose to impose more structure on the problem, as advocated by Just (2008) and Keane (2009). In order to do so, it may then be necessary to estimate principal-agent models using structural econometric methods.

\section{Supplementary Material}

Supplementary material is available at the American Journal of Agricultural Economics online at www.oxfordjournals.org/ our_journals/ajae/.

\section{References}

Ackerberg, D. A., and M. Botticini. 2000. The Choice of Agrarian Contracts in Early 
Renaissance Tuscany: Risk Sharing, Moral Hazard, or Capital Market Imperfections? Explorations in Economic History 37: 241-57.

Ackerberg, D. A., and M. Botticini. 2002. Endogenous Matching and the Empirical Determinants of Contract Choice. Journal of Political Economy 110: 564-91.

Arrow, K. 1971. Essays in the Theory of Risk Bearing. Chicago: Markham Publishing.

Becker, B. 2006. Wealth and Executive Compensation. Journal of Finance 61: 379-97.

Bellemare, M. F. 2008. The (Im)Possibility of Reverse Share Tenancy. Working Paper, Duke University, Durham, NC.

Bolton, P., and M. Dewatripont. 2005. Contract Theory. Cambridge, MA: MIT Press.

Brown, Z. S., and M. F. Bellemare. 2009. The Structural Estimation of Principal-Agent Models by Least Squares. Working Paper, Duke University, Durham, NC.

Dubois, P. 2002. Moral Hazard, Land Fertility and Sharecropping in a Rural Area of the Philippines. Journal of Development Economics 68: 35-64.

Dubois, P., B. Jullien, and T. Magnac. 2008. Formal and Informal Risk Sharing in LDCs: Theory and Empirical Evidence. Econometrica 76: 679-725.

Fafchamps, M., and F. Gubert. 2007. The Formation of Risk Sharing Networks. Journal of Development Economics 83: 326-50.

Fafchamps, M., and S. Lund. 2003. Risk Sharing Networks in Rural Philippines. Journal of Development Economics 71: 261-87.

Fukunaga, K., and W. E. Huffman. 2009. The Role of Risk and Transaction Costs in Contract Design: Evidence from Farmland Lease Contracts in US Agriculture. American Journal of Agricultural Economics 91: 237-49.

Guo, M., and H. Ou-Yang. 2006. Incentives and Performance in the Presence of Wealth Effects and Endogenous Risk. Journal of Economic Theory 129: 150-91.
Holt, C. A., and S. K. Laury. 2002. Risk Aversion and Incentive Effects. American Economic Review 92: 1644-55.

Just, R. E. 2008. Distinguishing Preferences from Perceptions for Meaningful Policy Analysis. Presidential address presented at the AAEA annual meetings, Orlando, FL, 27-29 July.

Kahneman, D., and A. Tversky. 1979. Prospect Theory: An Analysis of Decision under Risk. Econometrica 47: 263-92.

Keane, M. P. 2009. Structural vs. Atheoretic Approaches to Economics. Journal of Econometrics forthcoming.

Kimball, M. 1990. Precautionary Savings in the Small and in the Large. Econometrica 58: 53-73.

Laffont, J. -J., and M. S. Matoussi. 1995. Moral Hazard, Financial Constraints and Sharecropping in El Oulja. Review of Economic Studies 62: 381-99.

Ligon, E. 1998. Risk Sharing and Information in Village Economies. Review of Economic Studies 65: 847-64.

Lybbert, T. J., and D. R. Just. 2007. Is Risk Aversion Really Correlated with Wealth? How Estimated Probabilities Introduce Spurious Correlation. American Journal of Agricultural Economics 89: 964-79.

Mas-Colell, A., M. D. Whinston, and J. R. Green. 1995. Microeconomic Theory. Oxford: Oxford University Press.

Menezes, C. F., and D. L. Hanson. 1970. On the Theory of Risk Aversion. International Economic Review 11:481-87.

Meyer, D. J., and J. Meyer. 2005. Relative Risk Aversion: What Do We Know? Journal of Risk and Uncertainty 31: 243-62.

Rogerson, W. P. 1985. The First-Order Approach to Principal-Agent Problems. Econometrica 53: 1357-68.

Townsend, R. M. 1994. Risk and Insurance in Village India. Econometrica 62: 539-91.

Zeckhauser, R., and E. Keeler. 1970. Another Type of Risk Aversion. Econometrica 38: 661-65. 